\title{
The complex gradient inequality with parameter
}

\author{
Maciej P. Denkowski ${ }^{1}$
}

Received: 3 December 2016 / Accepted: 3 November 2017 / Published online: 13 November 2017

C The Author(s) 2017. This article is an open access publication

\begin{abstract}
We prove that given a holomorphic family of holomorphic functions with isolated singularities at the origin and constant Milnor number, it is possible to obtain the gradient inequality with a uniform exponent.
\end{abstract}

Keywords Gradient inequality · Holomorphic functions · Branched coverings · Milnor number

Mathematics Subject Classification 32S05 - 14B05 - 32A10

\section{Introduction}

This note was inspired by a recent article of Płoski [9] concerning the semi-continuity of the Łojasiewicz exponent in a family of multiplicity-constant deformation of a finite holomorphic germ. Actually, our main tool is a method of Płoski used already in [8]. Of course, it would probably be possible to derive the main result presented herein from this article, but it seems interesting to give a direct, elementary and self-consistent proof, since it is a starting point for tackling a much more general problem.

We are interested in the complex Łojasiewicz Gradient Inequality: given a holomorphic function $f$ of $m$ variables and such that $f(0)=0$ it is possible to find an exponent $\theta \in(0,1)$ such that in a neighbourhood of the origin

Communicated by A. Constantin.

$\square \quad$ Maciej P. Denkowski

maciej.denkowski@uj.edu.pl

1 Institute of Mathematics, Faculty of Mathematics and Computer Science, Jagiellonian University, ul. Łojasiewicza 6, 30-348 Kraków, Poland 


$$
|f(x)|^{\theta} \leq \text { const. }\|\nabla f(x)\|
$$

where $\nabla f(x)$ denotes the complex gradient of $f$. The classical version of this inequality — for a real analytic function — was obtained by Łojasiewicz from his famous Łojasiewicz inequality that he used to solve L. Schwartz's Division Problem. One of the simplest and most important applications of this inequality is the study of the analytic gradient dynamics, see [7]. There the fact that $\theta \in(0,1)$ is of utmost importance. To make the paper self-consistent, we give a simple proof of this in our case in Sect. 2.

Our particular aim is to obtain a parameter version of this inequality, i.e. to prove that given a well-parametrized family of functions we can find (locally) a uniform exponent for this inequality to be satisfied by each of these functions. Probably the simplest case is presented in this paper and it concerns a $\mu$-constant holomorphic unfolding. It may be particularly interesting e.g. in view of the results in [4].

A general subanalytic parameter version of the gradient inequality will be given in a forthcoming paper.

\section{Preliminaries}

For the convenience of the reader we gather in this section all the basic notation and notions used throughout the paper.

\section{Order of vanishing.}

We denote by ord ${ }_{0} h$ the order of vanishing of the holomorphic germ $h \in \mathcal{O}_{m}$ at the origin, i.e. the degree of the initial form in the expansion of $h \not \equiv 0$ into homogeneous forms near the origin.

There is $\operatorname{ord}_{0} h=\max \left\{\eta>0|| h(z) \mid \leq\right.$ const. $\left.|| z\right|^{\eta}$ in a neighbourhood of 0$\}$, see [2]. For $h=\left(h_{1}, \ldots, h_{n}\right)$ with $h_{j} \in \mathcal{O}_{m}$, we put $\operatorname{ord}_{0} h=\min _{j} \operatorname{ord}_{0} h_{j}$.

From the expansion into a power series it is easy to see that for a non-constant analytic germ $g:(\mathbb{C}, 0) \rightarrow(\mathbb{C}, 0)$ there is $c_{1}|t|^{q} \leq|g(t)| \leq c_{2}|t|^{q}$ in a neighbourhood of the origin with $q=\operatorname{ord}_{0} g, c_{1}, c_{2}>0$. Hence, if $g=\left(g_{1}, \ldots, g_{n}\right)$ on $(\mathbb{C}, 0)$ the same kind of inequality holds true with $q=\min _{j} \operatorname{ord}_{0} g_{j}=\operatorname{ord}_{0} g$.

\section{Branched covering and geometric multiplicity.}

Given a finite holomorphic map germ $H:\left(\mathbb{C}^{m}, 0\right) \rightarrow\left(\mathbb{C}^{m}, 0\right)$ we can find a connected neighbourhood $U$ of $0 \in \mathbb{C}^{m}$ for which $H^{-1}(0) \cap U=H^{-1}(0) \cap \bar{U}=\{0\}$. This implies that $H$ ia proper map from $U$ onto a connected neighbourhood $V$ of the origin. This means that the projection $\pi: \mathbb{C}^{m} \times \mathbb{C}^{m} \ni(z, w) \mapsto w \in \mathbb{C}^{m}$ is proper on $\Gamma_{H} \cap(U \times V)$ where $\Gamma_{H}$ denotes the graph of $H$. It is thence a branched covering (see [2]) with a covering number (sheet number) called also geometric multiplicity that we will denote by $m(H)$. In this situation we call $H$ itself a branched covering. We denote by $\sigma_{H}$ its critical locus, i.e. the set of points $w \in V$ for which $\# H^{-1}(w)<m(H)$-in this case it coincides with the critical values in the usual sense.

Since each fibre is finite and the fibres vary continuously, we may define a geometric multiplicity at a point $z_{0} \in U$ as the generic number of points converging to $z_{0}$ from nearby fibres. We denote it by $m_{z_{0}}(H)$. In other words, if we fix a neighbourhood 
$W \subset U$ of $z_{0}$ such that $H^{-1}\left(H\left(z_{0}\right)\right) \cap \bar{W}=\left\{z_{0}\right\}, m_{z_{0}}(H)$ is the covering number of the branched covering $\left.H\right|_{W}$. In particular, $m(H)=m_{0}(H)$ in our situation.

It is a classical result due to W. Stoll that for any branched covering $H$ between domains in $\mathbb{C}^{m}$,

$$
m(H)=\sum_{z \in H^{-1}\left(H\left(z_{0}\right)\right)} m_{z}(H) .
$$

\section{Characteristic polynomial.}

Given a holomorphic map germ $g:\left(\mathbb{C}^{m}, 0\right) \rightarrow\left(\mathbb{C}^{m}, 0\right)$ satisfying $g^{-1}(0)=\{0\}$ and any holomorphic germ $f \in \mathcal{O}_{m}$ we can define the characteristic polynomial of $f$ with respect to $g$ in the following way. The map $g$ defines a branched covering between domains $U, V \subset \mathbb{C}^{m}$. Let $\mu=m(g)$ be its covering number and $\sigma$ its critical locus. Then for $w \in V \backslash \sigma$ we have $g^{-1}(w)=\left\{z^{(1)}, \ldots, z^{(\mu)}\right\}$ consisting of exactly $\mu$ points and so we can define for $t \in \mathbb{C}$

$$
\begin{aligned}
P(w, t): & =\prod_{z \in g^{-1}(w)}(t-f(z)) \\
& =t^{\mu}+a_{1}(w) t^{\mu-1}+\cdots+a_{\mu}(w), \\
& \text { where } a_{j}(w)=(-1)^{j} \sum_{1 \leq \iota_{1}<\cdots<\iota_{j} \leq \mu} f\left(z^{\left(\iota_{1}\right)}\right) \cdots f\left(z^{\left(\iota_{j}\right)}\right)
\end{aligned}
$$

are holomorphic functions on $V \backslash \sigma$ admitting continuous extensions onto $V$. Therefore, by the Riemann Extension Theorem, $P \in \mathcal{O}(V)[t]$. This polynomial is called the characteristic polynomial of $f$ with respect to $g$. Clearly, $P(g, f) \equiv 0$. Moreover, we have $a_{j}(0)=0$, provided $f(0)=0$.

\section{Effective gradient inequality.}

For convenience sake, we will give-using only complex analytic geometry tools in the spirit of Płoski-yet another and elementary proof of the gradient inequality for a holomorphic germ $f:\left(\mathbb{C}^{m}, 0\right) \rightarrow(\mathbb{C}, 0)$ satisfying the condition $\operatorname{dim}_{0} \nabla f^{-1}(0)=0$, where

$$
\nabla f=\left(\frac{\partial f}{\partial z_{1}}, \ldots, \frac{\partial f}{\partial z_{m}}\right): \mathbb{C}_{z}^{m} \rightarrow \mathbb{C}_{w}^{m}
$$

is the complex gradient. What will be of greatest interest for our purposes is the effective exponent we obtain.

We have the following Proposition (cf. [11] Corollaire 2 p. 270 and [8, 10]):

Proposition 2.1 If $f \in \mathcal{O}_{m}$ is a holomorphic germ such that $\nabla f$ has an isolated zero at the origin, then the coefficients of the characteristic polynomial $P(w, t)=$ $t^{\mu}+a_{1}(w) t^{\mu-1}+\cdots+a_{\mu}(w) \in \mathcal{O}_{m}[t]$, of $f$ with respect to $\nabla f$, where $\mu$ is the Milnor number of $f$, all satisfy $\operatorname{ord}_{0} a_{j} \geq j+1$ and we have in a neighbourhood of 
the origin the gradient inequality

$$
|f(z)|^{\max _{j=1}^{\mu} \frac{j}{\operatorname{ord}_{0} a_{j}}} \leq \text { const. }|| \nabla f(z) \| .
$$

Proof We may assume $m>1$, since the case $m=1$ is a simple exercise involving power series. Our approach is based on [8].

Take a connected neighbourhood $U$ of $0 \in \mathbb{C}^{m}$ for which $\nabla f^{-1}(0) \cap U=$ $\nabla f^{-1}(0) \cap \bar{U}=\{0\}$. Hence for some neighbourhood $V$ of the origin, $\nabla f: U \rightarrow V$ is a branched covering with covering number $\mu=m_{0}(\nabla f)$ that is in fact the Milnor number of $f$ at the origin. Let $P \in \mathcal{O}(V)[t]$ the characteristic polynomial of $f$ with respect to $\nabla f$. It is a distinguished Weierstrass polynomial (cf. the Viète formulæ for the coefficients and the fact that $f$ vanishes at the origin).

For the convenience of the reader let us recall the following easy, but apparently forgotten inequality:

Lemma 2.2 If $t_{0}$ is a root of the unitary polynomial $p(t)=t^{\mu}+\sum_{j=1}^{\mu} a_{j} t^{\mu-j} \in \mathbb{C}[t]$, then $\left|t_{0}\right| \leq 2 \max _{j=1}^{\mu}\left|a_{j}\right|^{1 / j}$.

Proof Suppose that $\left|t_{0}\right|>2 \max _{j=1}^{\mu}\left|a_{j}\right|^{1 / j}$, i.e. $\left|a_{j}\right|<\left|t_{0}\right|^{j} / 2^{j}$, for each $j=$ $1, \ldots, \mu$. From $p\left(t_{0}\right)=0$ we obtain

$$
\begin{aligned}
\left|t_{0}\right|^{\mu} & =\left|\sum_{j=1}^{\mu} a_{j} t_{0}^{\mu-j}\right| \\
& \leq \sum_{j=1}^{\mu}\left|a_{j}\right| \cdot\left|t_{0}\right|^{\mu-j}<\sum_{j=1}^{\mu} \frac{\left|t_{0}\right|^{j}}{2^{j}}\left|t_{0}\right|^{\mu-j} \\
& \leq\left|t_{0}\right|^{\mu} \sum_{j=1}^{+\infty} \frac{1}{2^{j}}=\left|t_{0}\right|^{\mu}
\end{aligned}
$$

which is a contradiction.

In our case, for a fixed $z, f(z)$ is a root of the polynomial $t \mapsto P(\nabla f(z), t)$, whence by the Lemma above, $|f(z)| \leq 2 \max _{j}\left|a_{j}(\nabla f(z))\right|^{1 / j}$. On the other hand, since in a neighbourhood of the origin there is $\left|a_{j}(w)\right| \leq c_{j}|| w \|^{\text {ord }_{0} a_{j}}$, for some $c_{j}>0$, we can write

$$
\left|a_{j}(\nabla f(z))\right|^{1 / j} \leq c_{j}^{1 / j}\|\nabla f(z)\|^{\frac{\operatorname{ord}_{0} a_{j}}{j}},
$$

for all $j=1, \ldots, \mu$ and all points $z$ in a neighbourhood of the origin, because the gradient vanishes at the origin. In particular, we may assume that in this neighbourhood $\|\nabla f(z)\|<1$. Eventually, all these inequalities lead to

$$
|f(z)| \leq \text { const. }\|\nabla f(z)\|^{\min _{j=1}^{\mu}\left(\frac{\operatorname{ord}_{0} a_{j}}{j}\right)}
$$


in a neighbourhood of the origin. We have obtained the gradient inequality with the exponent

$$
\theta=\max _{j=1}^{\mu} \frac{j}{\operatorname{ord}_{0} a_{j}}
$$

and we shall prove yet that $\theta<1$, i.e. $\operatorname{ord}_{0} a_{j}>j$ for all $j$. This is still rather delicate (see the crucial article [11] of B. Teissier, Corollaire 2 p. 270 and compare with [10] Theorem 2).

Recall the Peano tangent cone:

$$
C_{0}\left(P^{-1}(0)\right)=\left\{v \in \mathbb{C}^{m+1} \mid \exists P^{-1}(0) \ni x_{v} \rightarrow 0, \exists \lambda_{v}>0: \lambda_{v} x_{v} \rightarrow v\right\} .
$$

It is described by the zeroes of the initial form in $P$ in the expansion of $P$ into homogeneous forms at the origin. Therefore, $\theta<1$ being equivalent to in $P(w, t)=t^{\mu}$, we have to show that $C_{0}\left(P^{-1}(0)\right)=\mathbb{C}^{m} \times\{0\}$. This amounts to showing that $\mathbb{C}^{m} \times\{0\} \supset C_{0}\left(P^{-1}(0)\right)$, for we know that $C_{0}\left(P^{-1}(0)\right)$ is an algebraic cone of dimension $\operatorname{dim}_{0} P^{-1}(0)=m$.

First, we will show that $\{0\}^{m} \times \mathbb{C} \cap C_{0}\left(P^{-1}(0)\right)=\{0\}^{m+1}$. It is well-known that $f$ is integral over the ideal generated by $z_{j} \frac{\partial f}{\partial z_{j}}, j=1, \ldots, m$ in $\mathcal{O}_{m}\left({ }^{1}\right)$ and so from the equation of integral dependence we obtain the inequality $|f(z)| \leq$ const. ||$\nabla f(z) \|$ in a neighbourhood of the origin (see e.g. [1]). Now, since $P^{-1}(0)$ and $(\nabla f, f)(U)$ define the same set germ at the origin, we see that for any $v=(0, t) \in C_{0}\left(P^{-1}(0)\right)$ we can find sequences $U \ni z_{v} \rightarrow 0$ and $\lambda_{v} \rightarrow 0^{+}$such that $\lambda_{v} \nabla f\left(z_{v}\right) \rightarrow 0$ and $\lambda_{\nu} f\left(z_{v}\right) \rightarrow t$. Therefore, we get $t=0$.

Shrinking the neighbourhoods we may assume that we have $P^{-1}(0)=(\nabla f, f)(U)$ and this has pure dimension $m$. Then, in view of the transversality just proved, we can use the elementary Proposition 1 from [2] 8.5 (p. 86): for any $(w, t) \in C_{0}\left(P^{-1}(0)\right) \backslash$ $\{(0,0)\}$, in particular for $w \neq 0$, we have

$$
(w, t) \in C_{0}\left(P^{-1}(0) \cap(\mathbb{C} w \times \mathbb{C})\right) .
$$

But, $P^{-1}(0) \cap(\mathbb{C} w \times \mathbb{C})=\left\{(\nabla f(z), f(z)) \mid z \in \nabla f^{-1}(\mathbb{C} w)\right\}$ is a curve as the image by $(\nabla f, f)$ of the polar curve $X:=\nabla f^{-1}(\mathbb{C} w)(\nabla f$ is surjective and we are in a neighbourhood of the origin). Fix $(w, t)$ and denote by $\Gamma$ an irreducible component of $(\nabla f, f)(X)$ for which $(w, t) \in C_{0}(\Gamma)$. Then there exists $v \in \mathbb{C}^{m+1} \backslash\{0\}$ such that $C_{0}(\Gamma)=\mathbb{C} v$.

Let $\Gamma^{\prime} \subset X$ be an irreducible component such that $(\nabla f, f)\left(\Gamma^{\prime}\right)=\Gamma$. Consider a Puiseux parametrization $\gamma: W \rightarrow \Gamma^{\prime}$. Then $h:=(\nabla f, f) \circ \gamma$ has image $\Gamma$ and so by the definition of the tangent cone there is a sequence $t_{v} \rightarrow 0$ such that $v=$ $\lim h\left(t_{v}\right) /\left\|h\left(t_{v}\right)\right\|$.

\footnotetext{
1 This can be easily checked using the valuation criterion proved by Lejeune-Jalabert and Teissier in [6] and cited in [1].
} 
Write $v=\left(v^{\prime}, v^{\prime \prime}\right) \in \mathbb{C}^{m} \times \mathbb{C}$; we will show that $v^{\prime \prime}=0$. We look at $f\left(\gamma\left(t_{v}\right)\right)$; on the one hand,

$$
\left|f\left(\gamma\left(t_{v}\right)\right)\right| \leq \text { const. }\left|t_{v}\right|^{\operatorname{ord}_{0}(f \circ \gamma)}, v \gg 1,
$$

whereas on the other,

$$
\left\|h\left(t_{v}\right)\right\| \geq\left\|\nabla f\left(\gamma\left(t_{v}\right)\right)\right\| \geq \text { const. }\left|t_{v}\right|^{\operatorname{ord}_{0}(\nabla f \circ \gamma)}, v \gg 1
$$

where $\operatorname{ord}_{0}(\nabla f \circ \gamma)=\min _{j} \operatorname{ord}_{0}\left(\frac{\partial f_{j}}{\partial z} \circ \gamma\right)$. For $v$ large enough,

$$
\frac{\left|f\left(\gamma\left(t_{v}\right)\right)\right|}{|| h\left(t_{v}\right)||} \leq \text { const. }\left|t_{v}\right|^{\operatorname{ord}_{0}(f \circ \gamma)-\operatorname{ord}_{0}(\nabla f \circ \gamma)}
$$

and it remains to observe that the exponent is positive.

To see this we argue classically as follows: if $g:(\mathbb{C}, 0) \rightarrow(\mathbb{C}, 0)$ is an analytic germ, then $\operatorname{ord}_{0} g=\operatorname{ord}_{0} g^{\prime}+1$, whence $\operatorname{ord}_{0}(f \circ \gamma)=\operatorname{ord}_{0}(f \circ \gamma)^{\prime}+1$. But since $\operatorname{ord}_{0} \sum_{1}^{k} g_{j} \geq \min _{j} \operatorname{ord}_{0} g_{j}$,

$$
\begin{aligned}
\operatorname{ord}_{0}(f \circ \gamma)^{\prime} & =\operatorname{ord}_{0}\left\langle\nabla f \circ \gamma, \gamma^{\prime}\right\rangle \\
& \geq \min _{j} \operatorname{ord}_{0}\left(\frac{\partial f}{\partial z_{j}} \circ \gamma\right) \gamma_{j}^{\prime} \\
& =\min _{j}\left\{\operatorname{ord}_{0}\left(\frac{\partial f}{\partial z_{j}} \circ \gamma\right)+\operatorname{ord}_{0} \gamma_{j}^{\prime}\right\} .
\end{aligned}
$$

Since $\operatorname{ord}_{0} \gamma_{j}^{\prime} \geq 0$ for all $j$, the last expression is greater or equal to $\operatorname{ord}_{0}(\nabla f \circ \gamma)$.

\section{Gradient inequality with parameter}

Let $f(x, t) \in \mathcal{O}_{m+k}$ be a holomorphic germ. Write $f_{t}(x):=f(x, t)$ and put $g_{t}(x):=$ $\nabla f_{t}(x)$ for the complex gradient. The resulting germ $g(x, t):=g_{t}(x)$ is, of course, holomorphic. Throughout this section we assume that $f(0, t) \equiv 0$ (which is not really restrictive).

We will need an auxiliary result that should be compared with condition (6) from the Lê-Saito-Teissier criterion of $\mu$-constancy for one-parameter unfoldings as exposed in [3] p. 161 (compare also the principle of the conservation of the total Milnor number in [5]):

Proposition 3.1 Keeping the notations introduced above, assume that $f \in \mathcal{O}_{m+k}$ is such that $g_{t}(0)=0$ for $|t| \ll 1$ and that $g_{0}^{-1}(0)=\{0\}$ is isolated. Then

(1) For all t sufficiently close to zero, $g_{t}^{-1}(0)$ is isolated too and the resulting branched coverings are all $\mu$-sheeted, where $\mu=m_{0}\left(g_{0}\right)$ is the Milnor number of $g_{0}$; in particular the Milnor numbers of $g_{t}$ are not greater than $\mu$; 
(2) The unfolding $f$ of $f_{0}$ is $\mu$-constant iff in a neighbourhood of the origin we have

$$
\left\{(x, t) \in \mathbb{C}^{m} \times \mathbb{C}^{k}: \frac{\partial f}{\partial x_{j}}(x, t)=0, j=1, \ldots, m\right\}=\{0\}^{m} \times \mathbb{C}^{k} .
$$

Proof If we assume that $g_{0}^{-1}(0)=\{0\}$ is isolated, then the holomorphic germ $G(x, t):=(g(x, t), t)$ has an isolated zero at the origin, since $G^{-1}(0)=g_{0}^{-1}(0) \times\{0\}$. Therefore, we may consider it as a branched covering between domains that can be chosen to be polydiscs, $U \times V, W \subset \mathbb{C}^{m+k}$ centred at the origin, $G$ having geometric multiplicity $m(G)$ coinciding with the geometric multiplicity $m_{0}(G)$ at the origin. Put $\mu:=m_{0}(G)$.

Observe that $G^{-1}(y, t)=g_{t}^{-1}(y) \times\{t\}$ and in particular all the $g_{t}, t \in V$, are branched coverings $U \rightarrow W_{t}$, where $W_{t}=\left\{x \in \mathbb{C}^{m} \mid(x, t) \in W\right\}$. However, by (\#) in the Preliminaries, $m\left(g_{t}\right) \geq m_{0}\left(g_{t}\right)$ where the latter is precisely the Milnor number of $f_{t}$, since $g_{t}(0)=0$.

Clearly, $m\left(g_{t}\right) \leq \mu$. In order to see that $m\left(g_{t}\right)=\mu$, for all $t \in V$, we will look first at the critical locus $\sigma$ of $G$, which is precisely $G\left(C_{G}\right)$ where $C_{G}=\{(x, t) \in U \times V \mid$ $\left.\operatorname{det} d_{(x, t)} G=0\right\}$ is the set of critical points. Observe that

$$
\operatorname{det} d_{(x, t)} G=\operatorname{det} \frac{\partial g}{\partial x}(x, t)=\operatorname{det} \frac{\partial g_{t}}{\partial x}(x) .
$$

But we know that the critical locus $\sigma_{t}$ of $g_{t}$ is precisely $g_{t}\left(C_{g_{t}}\right)$ and so we conclude that

$$
x \in \sigma_{t} \Leftrightarrow(x, t) \in \sigma .
$$

In particular, this implies that the sections $\sigma \cap\left(\mathbb{C}^{m} \times\{t\}\right)$ are nowheredense in $W \cap$ $\left(\mathbb{C}^{m} \times\{t\}\right)$ whence we conclude that $m\left(g_{t}\right)=\mu$ for all $t \in V$. Indeed, for each $t$, there is always a point $y$ near zero that is critical neither for $G$ (i.e. $(y, t) \notin W \backslash \sigma$ ), nor for $g_{t}$ (i.e. $y \notin \sigma_{t}$ ) and so

$$
\mu=\# G^{-1}(y, t)=\#\left(g_{t}^{-1}(y) \times\{t\}\right)=\# g_{t}^{-1}(y)=m\left(g_{t}\right) .
$$

The first part of the assertion is proved. The second part is again a consequence of (\#). Indeed, we already know that $m\left(g_{t}\right)=\mu$ and we have $g_{t}(0)=0$. Thus, the formula in question implies that $m_{0}\left(g_{t}\right)=\mu$ iff $\# g_{t}^{-1}(0)=1$. This gives (2).

For the rest of the section we assume as in the result above that for all $t$ small enough, $g_{t}^{-1}(0)=\{0\}$ is isolated at the origin. Furthermore, we assume that the Milnor numbers $m_{0}\left(g_{t}\right) \equiv \mu$ are constant. Then our main theorem is the following:

Theorem 3.2 If $f(x, t) \in \mathcal{O}_{m+k}$ is such that for all $t$ small enough, $f_{t}(x)$ has an isolated singularity at the origin of $\mathbb{C}^{m}$ with constant Milnor number $\mu$, then for all $(x, t)$ in a neighbourhood of the origin in $\mathbb{C}^{m+k}$,

$$
\left|f_{t}(x)\right|^{\frac{\mu}{\mu+1}} \leq C|| \nabla f_{t}(x) \|
$$

where $C>0$. 
Remark 3.3 Note that the exponent belongs to $[1 / 2,1)$ and $f$ itself satisfies the gradient inequality (with respect to $(x, t))$ with this exponent.

Proof of Theorem 3.2 The proof will be given in several steps.

\section{Step 1}

Consider as in the preceding Proposition the proper mapping germ $G(x, t):=$ $(g(x, t), t), G:\left(\mathbb{C}^{m+k}, 0\right) \rightarrow\left(\mathbb{C}^{m+k}, 0\right)$.

By Proposition 3.1 we know that the multiplicity at the origin of the branched covering $G$ is $\mu$.

\section{Step 2}

Let now $P(y, t, s) \in \mathcal{O}_{m+k}[s]$ be the characteristic polynomial of $f$ with respect to $G$. Therefore, we have $P(t, g(x, t), f(x, t)) \equiv 0$ and so by the Viète formulæ:

$$
|f(x, t)| \leq 2 \max _{j=1}^{\mu}\left|a_{j}(g(x, t), t)\right|^{1 / j}
$$

where we write $P(y, t, s)=s^{\mu}+a_{1}(y, t) s^{\mu-1}+\cdots+a_{\mu}(y, t)$.

Lemma 3.4 $P(\cdot, t, \cdot)$ is the characteristic polynomial of $f_{t}$ with respect to $g_{t}$.

Proof For a generic point $y$ near zero, there is $\# g_{t}^{-1}(y)=\mu$. Let $x^{(1)}, \ldots, x^{(\mu)}$ be the points in this fibre. Then since the fibre $G^{-1}(y, t)$ consists precisely of the points $\left(x^{(j)}, t\right)$, it is maximal and we get

$$
P(y, t, s)=\prod_{j=1}^{\mu}\left(s-f\left(x^{(j)}, t\right)\right)=\prod_{j=1}^{\mu}\left(s-f_{t}\left(x^{(j)}\right)\right)
$$

which gives the assertion.

In particular we have $a_{j}(0, t)=0$.

\section{Step 3}

Fix $j$ and consider the expansion of $a_{j}$ into a Hartogs series in a polydisc $P(r)$ centred at zero (the same for all $j=1, \ldots, \mu$ )

$$
a_{j}(y, t)=\sum_{\nu=v_{j}(t)}^{+\infty} \sum_{\substack{\alpha \in \mathbb{Z}_{+}^{m} \\|\alpha|=v}}\left(\frac{1}{\alpha !} D^{(\alpha, 0)} a_{j}(0, t)\right) y^{\alpha}
$$

where $v_{j}(t):=\operatorname{ord}_{0} a_{j}(\cdot, t) \geq j+1$ by Proposition 2.1. Write

$$
b_{j v}^{t}(y)=\sum_{\substack{\alpha \in \mathbb{Z}_{+}^{m} \\|\alpha|=v}}\left(\frac{1}{\alpha !} D^{(\alpha, 0)} a_{j}(0, t)\right) y^{\alpha}
$$


for the $\nu$-homogeneous term. For $(y, t) \in P(r)$ fixed and $\lambda \in \mathbb{C}$ we have

$$
a_{j}\left(\frac{y}{\|y\|} \lambda, t\right)=\sum_{\nu=v_{j}(t)}^{+\infty}\left(\frac{b_{j \nu}^{t}(y)}{\|y\|^{\nu}}\right) \lambda^{\nu}, \text { provided }|\lambda|<r .
$$

The Cauchy inequalities yield

$$
\left|\frac{b_{j v}^{t}(y)}{\|y\|^{v}}\right| \leq \sup _{|\lambda|<r} \frac{\left|a_{j}\left(\frac{y}{\|y\|} \lambda, t\right)\right|}{r^{v}} \leq \frac{M_{j}}{r^{v}} .
$$

with $M_{j}:=\sup \left\{\left|a_{j}(y, t)\right|:(y, t) \in P(r)\right\}>0$. Let $M=\max _{j=1}^{\mu} M_{j}$. Writing $j+1$ instead of $v_{j}(t)$ we have in $P(r)$,

$$
\begin{aligned}
\left|a_{j}(y, t)\right| & \leq \sum_{\nu=j+1}^{+\infty} \frac{M}{r^{\nu}}\|y\|^{\nu} \\
& \leq\|y\|^{j+1} \frac{M}{r^{j+1}} \sum_{\nu=0}^{+\infty}\left(\frac{\|y\|}{r}\right)^{\nu} .
\end{aligned}
$$

This leads to the conclusion that for some constant $C>0$ we have for all $(y, t)$ in a neighbourhood $V$ of the origin and all indices $j$,

$$
\left|a_{j}(y, t)\right| \leq C|| y \|^{j+1}
$$

\section{Step 4}

Note that $g(0, t)=0$ and we obtain the inequalities

$$
\left|a_{j}(g(x, t), t)\right| \leq\left. C|| g(x, t)\right|^{j+1}
$$

for all $j$ and $(x, t)$ in a neighbourhood $U$ of the origin such that $G(U) \subset V$.

This gives the gradient inequality with parameter and

$$
\max _{j=1}^{\mu} \frac{j}{j+1}=\frac{\mu}{\mu+1}
$$

as exponent. The proof is complete.

Remark 3.5 In view of the preceding discussion, if the unfolding is not $\mu$-constant, we have at least the inequality $\mu_{t} \leq \mu_{0}$ for $t$ close to zero, where $\mu_{t}=m_{0}\left(g_{t}\right)$ is the Milnor number of $f_{t}$. Since the function $s \mapsto \frac{s}{s+1}$ is increasing and we may always assume that we are in a neighborhood in which $|f(t, x)|<1$, we easily obtain that

$$
\left|f_{t}(x)\right|^{\frac{\mu_{0}}{\mu_{0}+1}} \leq C_{t}\left\|\nabla f_{t}(x)\right\|
$$


in a neighbourhood $V_{t}$ of the origin in $\mathbb{C}^{m}$ that will depend on $t$ (cf. Proposition 3.1) just as the constant $C_{t}$.

Acknowledgements This research was partially supported by Polish Ministry of Science and Higher Education Grant 1095/MOB/2013/0. The author would like to thank A. Płoski for the reference [11] and M. Tibăr for attracting his attention to the paper [3]. Many thanks are due to the University of Lille 1 for excellent working conditions. The author is indebted to the anonymous referee for suggestions that improved the readability of the paper.

Open Access This article is distributed under the terms of the Creative Commons Attribution 4.0 International License (http://creativecommons.org/licenses/by/4.0/), which permits unrestricted use, distribution, and reproduction in any medium, provided you give appropriate credit to the original author(s) and the source, provide a link to the Creative Commons license, and indicate if changes were made.

\section{References}

1. Briançon, J., Skoda, H.: Sur la clôture intégrale d'un idéal de germes de fonctions holomorphes en un point de $\mathbb{C}^{n}$. C. R. Acad. Sci. Paris Sér. A 278, 949-951 (1974)

2. Chirka, E.M.: Complex Analytic Sets. Kluwer, Dordrecht (1989)

3. Greuel, G.-M.: Constant Milnor number implies constant multiplicity for quasihomogeneous singularities. Manuscr. Math. 56, 159-166 (1986)

4. Gaffney, T., Araújo dos Santos, R.: Real integral closure and Milnor fibrations. In: Real and Complex Singularities, London Mathematical Society Lecture Note Series, 380, Cambridge University Press, Cambridge, pp. 146-157 (2010)

5. Jiang, G., Tibăr, M.: Splitting of singularities. J. Math. Soc. Jpn. 54(2), 255-271 (2002)

6. Lejeune-Jalabert, M., Tessier, B., Risler, J.-J.: Clôture intégrale des idéaux et équisingularité, Ann. Toulouse Sér. 6, 17 no. 4 , 781-859, arXiv:0803.2369 (2008)

7. Łojasiewicz, S.: Sur les trajectoires du gradient d'une fonction analytique. Sem. di Geom. 1982-1983, Univ. di Bologna, pp. 115-117 (1983)

8. Płoski, A.: Multiplicity and the Łojasiewicz exponent. Banach Centre Publ. 20 - Singularities, Warsaw 1985, pp. 353-354 (1988)

9. Płoski, A.: Semicontinuity of the Łojasiewicz exponent. Univ. Iagel. Acta Math. No. 48, 103-110 (2010)

10. Płoski, A., Winiarski, T.: On a theorem of Platte. Bull. Polish Acad. Sci. 39(1-2), 57-61 (1991)

11. Teissier, B.: Variétés polaires I: Invariants polaires des singularités d'hypersurfaces. Invent. Math. 40, 267-292 (1977) 